\title{
Changes in Candidal Count Following Provision of Complete Denture
}

\author{
Momina Akram ${ }^{1}$ \\ Muhammad Waseem Ullah Khan ${ }^{2}$ \\ Sabiha Naeem ${ }^{3}$ \\ BDS, FCPS \\ BDS, FCPS \\ BDS
}

OBJECTIVE: Candida associated denture stomatitis is the most common oral disease in denture wearers. The density of Candida in individuals using prosthesis is very important in terms of infection development. As denture stomatitis is a multifactorial disease, so denture wearing, along with many other factors, involves in the changes of candidal count. Determine the candidal count after providing the patients with complete dentures as quantification of candidal count is helpful in differentiating between colonization and infection.

METHODOLOGY: This study was conducted in Department of Prosthodontics, Punjab Dental Hospital, Lahore. 155 edentulous patients visiting the Prosthodontic department were selected on the basis of inclusion and exclusion criteria. Before insertion of complete dentures, oral sample were taken using the oral rinse technique. The sample was inoculated on Sabouraud's agar, and incubated at 220C for $48 \mathrm{hrs}$. The number of colonies characteristic of Candida was counted. Denture hygiene instructions given. The patients were recalled after three months and the same oral sampling and culture technique were employed. RESULTS: The mean age of the patients that were included in the study was $57.97 \pm 4.99$ years. In this study majority of the patients were male and constituted almost $80 \%$ of the sample population. The mean colony forming units before provision of complete dentures were 19.75 with a standard deviation of 12.30 , this is considerably less than colony forming units after provision of complete dentures which was 165.37 with a standard deviation of 73.85 .

CONCLUSION: Denture wearing with meticulous oral hygiene in elderly individuals is of great concern in controlling candidal growth. Denture removal at night is recommended to help in eliminating the source of the infectious agent.

KEYWORDS: Candida, Denture Stomatitis, Culture Technique, Colony Forming Units.

HOW TO CITE: Akram M, Khan MWU, Naeem S. Changes in candidal count following provision of complete denture. J Pak Dent Assoc 2020;29(2):77-80.

DOI: https://doi.org/10.25301/JPDA.292.77

Received: 06 August 2019, Accepted: 13 January 2020

\section{INTRODUCTION}

$\mathrm{O}$ ral mucosal inflammation occurs in subjects carrying removable prosthesis and its most commonly diagnosed as Candida-associated denture stomatitis. ${ }^{1}$ Candida albicans is considered as the principle causative agent. Almost $60-70 \%$ of all complete denture wearers have denture stomatitis. ${ }^{2}$ Candidiasis is mainly manifested as a biofilm formation on the surfaces of prosthesis in which Candida is the main culprit. Residual microorganisms and debris accumulate on the irregular surface of the prosthesis even if it is cleaned thoroughly thus result in the continuous re-infection of the palate. ${ }^{3}$

1. Assistant Professor, Department of Prosthodontics, deMontmorency College of Dentistry Punjab Dental Hospital, Lahore.

2. Assistant Professor, Department of Prosthodontics, deMontmorency College of Dentistry Punjab Dental Hospital, Lahore.

3. FCPS Resident, Department of Prosthodontics, deMontmorency College of Dentistry Punjab Dental Hospital, Lahore.

Corresponding author: "Dr. Sabiha Naeem" < cute_sabi85@yahoo.com >
After wearing prosthetic appliances, mucosal damage occurs due to two main reasons i.e. Candidal colonization and proteinase secretion by the organisms (filamentous form). Factors such as biofilm formation and its adhesion on the surface of prosthesis, dimorphism, enzymes secretion i.e. proteinases and phospholipases, convert the Candida species into an effective pathogen. ${ }^{4}$ Other factors involve in its pathogenicity are AIDS, diabetes mellitus, head-neck cancer radiation therapy implementation, long-term antibiotic or corticosteroid use or nutritional disorders. Adhesion of Candida albicans occurs to acrylic surfaces of prosthesis and epithelial buccal cells than any other surfaces..$^{5,6}$

Use of complete dentures can also result in erythematous oral candidiasis. It is a pathological fungal condition manifested as reddish lesions with an atrophic appearance, it can be asymptomatic or symptomatic. In symptomatic condition, it is associated with mild burning sensation. ${ }^{7}$

This study is being conducted to determine the candidal count after providing the patients with complete dentures as 
quantification of candidal count is helpful in differentiating between colonization and infection. Appropriate denture management is important in controlling pathogenicity of Candida to promote general health.

\section{METHODOLGY}

This study was conducted in Department of Prosthodontics, Punjab Dental Hospital, Lahore. One hundred and fifty-five edentulous patients visiting the Prosthodontic department were selected on the basis of inclusion and exclusion criteria by Non-Probability, Consecutive Sampling.

Inclusion Criteria:

- New complete denture wearers.

- Healthy mucosa.

- Age > 50 years.

- Patient being edentulous for 06 months.

Exclusion Criteria:

- Diabetics.

- Smokers.

- Chemotherapy \& radiotherapy patients

- History of broad-spectrum antibiotics or steroid therapy in the past six months.

- Immunocompromised patients.

Informed written consent of the patient was taken after detailed verbal explanation of purpose, procedure, risk and the benefit to them.

Before insertion of complete dentures oral sample were taken using the oral rinse technique. (patient was asked to take a sip of distilled water, rinse for $30 \mathrm{sec}$ and spit) The sample was injected on Sabouraud's agar, and incubated at $220 \mathrm{C}$ for $48 \mathrm{hrs}$. The number of Candidal colonies was counted. Colony forming units (CFU) per milliliter were calculated (candidal counts $>400 \mathrm{CFU} / \mathrm{ml}$ were predictive of oral candidiasis). Germ tube tests were conducted to confirm the presence of Candida gram staining. Patients were provided with complete dentures. Minor adjustments of the denture were done if required. After the insertion, patients were instructed about denture hygiene verbally and written instructions were also given. Denture hygiene instructions were how to brush before sleep and after meals. The patients were recalled after three months and the same oral sampling and culture technique were employed.

\section{STATISTICAL ANALYSIS}

Data was analyzed through SPSS (version 10.0) software. The variables under study were age, gender and CFU (candidal colony forming units). Paired sample t-test was used to compare the candidal count before and after provision of complete dentures. P- value $>0.05$ was significant.

\section{RESULTS}

A total of 155 patients were enrolled in the study. The mean age of the patients that were included in the study was $57.97 \pm 4.99$ years (Figure 1). In this study out of 155 patients 123 were male and constituted almost $80 \%$ of the sample

Figure 1: Mean Age of the Patients

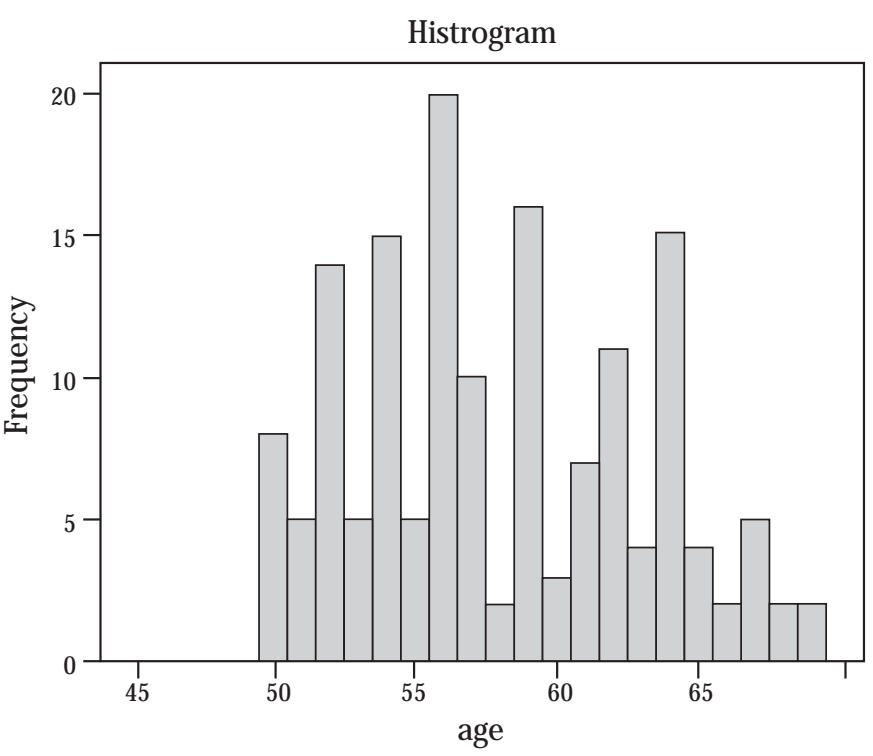

Table 1: Gender of the Patients

\begin{tabular}{|c|c|c|}
\hline & Frequency & Percentage \\
\hline Male & 123 & 79.4 \\
\hline Female & 32 & 20.6 \\
\hline Total & 155 & 100.0 \\
\hline
\end{tabular}

Table 2: Mean values for Cfu before \& Cfu after the provision of Complete Dentures. Cfu; Colony Forming Units

\begin{tabular}{|c|c|c|c|}
\hline & N & Minimum & Maximum \\
\hline Cfu before & 155 & 7 & 115 \\
\hline Cfu after & 155 & 39 & 394 \\
\hline
\end{tabular}

Table 3: Comparison between Colony Forming Units (CFU) before and after provision of Complete Dentures

\begin{tabular}{|l|c|c|c|c|c|}
\hline & Mean & $\mathrm{N}$ & $\begin{array}{c}\text { Std. } \\
\text { Deviation }\end{array}$ & $\begin{array}{c}\text { Std. Error } \\
\text { Mean }\end{array}$ & P-value \\
\hline $\begin{array}{l}\text { Cfu } \\
\text { before }\end{array}$ & 19.75 & 155 & 12.300 & .988 & $<0.0001$ \\
\hline \multirow{2}{\text{Cfuafter}}{} & 165.37 & 155 & 73.856 & 5.932 & \\
\hline
\end{tabular}


population. In contrast only 32 females were part of the study and just made $20.6 \%$ of whole sample (Table 1 ). Out of 155 patients, colony forming units exist in oral cavity before wearing dentures and fall in the range of 7-115. While after 3 months use of denture the range was 39-394 (Table 2).

The mean colony forming units before provision of complete dentures were 19.75 with a standard deviation of 12.30 , this is considerably less than colony forming units after provision of complete dentures which was 165.37 with a standard deviation of 73.85 (Table 3). Candidal count increased in all 155 patients after wearing of complete dentures for three months, however this increase did not exceed the cutt off value $400 \mathrm{CFU} / \mathrm{ml}$ to be declared as candidiasis.

Paired sample T-test was used to compare the candidal count before and after provision of complete dentures to the completely edentulous patients. The standard deviation was 68.76 and the standard error mean was 5.52, the confidence interval was kept at $95 \%$. P- value was less than 0.05 indicating there was a significant difference between the colony forming units before and after provision of complete dentures. This clearly indicates that there was significant rise in colony forming units after provision of complete dentures to the sample population.

\section{DISCUSSION}

Candida albicans, a microorganism exists in the oral floral community of healthy individuals either dentulous or edentulous. The oral cavity is a main reservoir which facilitates the colonization and spread of infection to systemic organs by pathogenic microorganisms. Oral candidiasis is developed due to the colonization of Candida species in response to long-term use of prosthesis. The density of Candida in denture wearers is very important factor in infection development. To specify the pathogenic species is important to start the proper treatment. Aging causes progressive increase in Candidal count in the oral cavity. ${ }^{7}$

The sole purpose of this study was to establish whether the wearing of dentures in the elderly patients results in increase in candidal count and whether that increase in candidal count is significant enough to be labeled as candidiasis. To provide reliable assessments to oral health, it is mandatory to account the stable and constant factor of denture wearing with its effects, which helps in observing the age or other data (in isolation) in quite easier way. Various species of yeasts have been implicated in the colonization of the oral cavity. ${ }^{8}$

However, studies have revealed that in patients with denture induced stomatitis, $62.1 \%$ of the isolated yeast belonged to Candida albicans species. Budtz-Jorgensen et al. (1975) and Makila and Hopsu-Havu (1977), both conducted their studies on denture wearers and demonstrated a high prevalence of yeasts in their sample population. Significant increase in candidal count even though statistically significant, however, was not sufficient enough to be labeled as candidiasis or candidal associated denture stomatitis. This is because cut-off point i.e. Candida counts $>400 \mathrm{CFU} / \mathrm{mL}$ of unstimulated saliva turns to $2 Æ 6$ after logarithmic transformation are predictive of oral candidiasis. ${ }^{9}$ In present study as well the cut off limit of $400 \mathrm{CFU} / \mathrm{ml}$ was used to define candidiasis. In this study the candidal count increased in all the 155 patients after provision and wearing of complete dentures for three months, however the increase in candidal count did not exceed the cut off limit of $400 \mathrm{CFU} / \mathrm{m} .^{1}$

Continuous denture wearing appears to facilitate the growth of species such as Candida albicans, Candida tropicalis, and Candida glabrata. ${ }^{1}$ Oral candidiasis is widely reported in elderly denture wearers. ${ }^{7,10}$ Several factors that govern the presence of C.albicans in the oral cavity are immunocompromised conditions e.g diabetes mellitus, AIDS, implementation of radiation therapy, nutritional disorders etc, and wearing dentures, long term antibiotic or steroid use, elderly people etc. It was for this reason that in this study we kept all the other factors constant and evaluated the effect of denture wearing on candidal count. Patients with any kind of systemic illnesses or condition that can modify the candidal count in the oral cavity were not included in the study and at the same time a specific age group was defined in which this study was conducted to minimize the effect that age can have in modifying the candidal count in the oral cavity. Schulman et al, reported that individuals wearing complete dentures for longer periods exhibited a higher candidal count as compared to people who used dentures for shorter periods and followed dentist's instructions for denture removal especially instructions regarding night time removal. According to Ikebe et al. if age, gender and drug intake are considered as controlling factors, then maxillary complete denture use is the only significant factor linked with candidal colonization in adults over 60 years. ${ }^{11}$

Salivary flow rate was not quantified in this study that was one of its limitations. As an overall assessment modality, saliva cultures are reliable. The errors in count was lessened by taking the sampling at the same time for every examination. Although continuous denture wearing was significant in Candida CFU counts but the denture-wearing habit alone is not enough to predispose the oral mucosa to the candidal infections. Various other factors are also involved in the fluctuations of Candida CFU counts such as age, gender, systemic heath, medications, quality and hygiene of the dentures and diet. ${ }^{8}$ In partial prosthesis users, severity of 
denture stomatitis is less as compare to complete denture wearers and fixed prosthesis users. This change in severity of disease is due to the change in the materials of prosthesis. As C.albicans is more adherent to acrylic surfaces and mucosa as compare to other surfaces. In fixed prosthesis mild stomatitis occurs because of the absence of acrylic material and also no mucosal contact exists. ${ }^{12.13}$ This study suggests that the candidal count increases after continuous denture wear and it is assessed as an important factor to predispose candidal infection. Thus, denture removal at night is advisable to eliminate the source of the pathogenicity and to maintain immaculate oral hygiene so as to minimize the risk of candidiasis in complete denture patients.

\section{CONCLUSION}

While giving oral hygiene guidance to the middle-aged patients, denture wearing habits must be considered as a significant factor. In elderly individuals, systemic heath is of utmost concern. Appropriate denture management is mandatory to promote general health by controlling Candidal colonization. In immunocompromised patients, Candidal colonization due to denture wearing is detrimental to patient's health. Microorganisms are entrapped in the irregular surface of acrylic resin especially Candida albicans. Therefore, to control the spread of candidal infection in risk patients who wear prostheses, immunosuppression is of critical importance.

\section{CONFLICTS OF INTEREST}

There are no conflicts of interest.

\section{SOURCE OF FUNDS}

There are no sources of funds involved in this study.

\section{REFERENCES}

1. Petrovic SM, Barac M, Pficer JK, Radunovic M, Jotic A, Pucar A. Presence of Different Candida Species at Denture Wearers with Type 2 Diabetes and Clinically Healthy Oral Mucosa-Pilot Study. Balk J Dent Med 2018;22:15-21. https://doi.org/10.2478/bjdm-2018-0003

2. Salerno C, Pascale M, Contaldo M, Esposito V, Busciolano M, Milillo L, Guida A, Petruzzi M, Serpico R. Candida-associated denture stomatitis. Med Oral Patol Oral Cir Bucal 2011;16:139-43. https://doi.org/10.4317/medoral.16.e139

3. Prakash B, Shekar M, Maiti B, Karunasagar I, Padiyath S. Prevalence of Candida spp. among healthy denture and nondenture wearers with respect to hygiene and age. J Indian Prosthodont Soc 2015;15:29-32. https://doi.org/10.4103/0972-4052.164912

JPDA Vol. 29 No. 02 Apr-Jun 2020
4. Gleiznys A, Zdanaviciene E, Žilinskas J. Candida albicans importance to denture wearers. A literature review. Stomatologija 2015;17:54-66.

5. Niaz A, Nayyer S. Candida albicans myocardial abscess. J Coll Physicians Surg Pak. 2003:456-58.

6. Lyu X, Zhao C, Yan ZM, Hua H. Efficacy of nystatin for the treatment of oral candidiasis: a systematic review and meta-analysis. Drug Des Dev Ther 2016;10:1161-171.

https://doi.org/10.2147/DDDT.S100795

7. Cankovic M, Bokor-Bratic M, Marinoski J, Stojanovic D. Prevalence and possible predictors of the occurrence of denture stomatitis in patients older than 60 years. Vojnosanitetski pregl 2017;74:311-16. https://doi.org/10.2298/VSP150104185C

8. Freitas JB, Gomez RS, De Abreu MH, Ferreira e Ferreira E. Relationship between the use of full dentures and mucosal alterations among elderly Brazilians. J Oral Rehabil 2008;35:370-74.

https://doi.org/10.1111/j.1365-2842.2007.01782.x

9. Marcos-Arias C, Eraso E, Madariaga L, Aguirre JM, Quindós G. Phospholipase and proteinase activities of Candida isolates from denture wearers. Mycoses 2011;54:10-6.

https://doi.org/10.1111/j.1439-0507.2009.01812.x

10. Dagistan S, Aktas AE, Caglayan F, Ayyildiz A, Bilge M. Differential diagnosis of denture-induced stomatitis, Candida, and their variations in patients using complete denture: a clinical and mycological study. Mycoses 2009;52:266-71.

https://doi.org/10.1111/j.1439-0507.2008.01592.x

11. Pires-Gonçalves RH, Miranda ET, Baeza LC, Matsumoto MT, Zaia JE, Mendes-Giannini MJ. Genetic relatedness of commensal strains of Candida albicans carried in the oral cavity of patients' dental prosthesis users in Brazil. Mycopathologia 2007;164:255-63. https://doi.org/10.1007/s11046-007-9052-5

12. White PL, Williams DW, Kuriyama T, Samad SA, Lewis MA, Barnes RA. Detection of candida in concentrated oral rinse cultures by real-time PCR. J Clin Micobiol 2004;42:2101-07. https://doi.org/10.1128/JCM.42.5.2101-2107.2004

13. Pires FR, Santos EB, Bonan PR, De Ameida OP, Lopez MA. Denture stomatitis and salivary candida in Brazilian edentulous patients. J Oral Rehabil 2002;29:115-19.

https://doi.org/10.1046/j.1365-2842.2002.00947.x 